\title{
Antimicrobial effect of aroma essential oils on the oral cavity for the prevention and treatment of inflammatory diseases.
}

\author{
Seoul-Hee Nam ${ }^{1}$, Mi-Sook Choi ${ }^{2}$, Young-Soon Choi ${ }^{3 *}$ \\ ${ }^{1}$ Department of Dental Hygiene, College of Health Science, Kangwon National University, Samcheok-si, Republic of \\ Korea \\ ${ }^{2}$ Department of Dental Hygiene, Andong Science College, Andong-si, Republic of Korea \\ ${ }^{3}$ Department of Nursing, College of Health Science, Kangwon National University, Samcheok-si, Republic of Korea
}

\begin{abstract}
The purpose of this study was to investigate the applicability of the next-generation antimicrobial materials, essential oils, as preventive and therapeutic agents for oral diseases. To determine the antimicrobial effect of five essential oils-lemon, tea tree, geranium, peppermint, and myrrh-against Candida albicans ( $C$. albicans), the diameters of the growth inhibition zones were measured. The study results showed that the five essential oils that were used in this study effectively inhibited the growth of bacteria, particularly $C$. albicans. The inhibition zones were $30 \mathrm{~mm}$ for lemon, $21 \mathrm{~mm}$ for myrrh, $20 \mathrm{~mm}$ for geranium, $19 \mathrm{~mm}$ for tea tree, and $19 \mathrm{~mm}$ for peppermint. Therefore, it is considered that essential oils can replace antibiotics and can lead to the promotion of oral health through the prevention and treatment of candidiasis in the dental field against $C$. albicans.
\end{abstract}

Keywords: Antimicrobial effect, Aroma essential oils, Oral candidiasis, Oral diseases, Candida albicans.

Accepted on December 11, 2018

\section{Introduction}

The lifestyle changes that have occurred since the start of the $21^{\text {st }}$ century due to industrialization and Westernization have led to the emergence of increasingly diverse oral diseases, which has emerged as a serious social problem [1]. A disease in the mouth is not only associated with the teeth or gums but also has a close relationship with the whole body's health, which has been steadily researched on [2].

Oral candidiasis is an oral infectious disease due to true fungi, which frequently occurs in the oral cavity of modern people [3]. It is an oral mucosal disease that occurs in the tongue, cheek, mucous membrane, or gums in the mouth. Candida albicans (C. albicans) is a typical organism that causes an opportunistic infection and is present as resident flora in the rubbed and wet skin and mucous membranes of the human body [4]. A major causative organism of oral candidiasis, $C$. albicans is present in the oral cavity of many people and exists without clinical symptoms of infection in the human oral cavity [5]. Since the 1990s, the number of opportunistic infections due to Candida has increased sharply as the number of patients treated with antibiotics or anticancer agents and the number of patients with endocrine imbalance and reduced salivary gland function increase [6].

There is a growing interest in oral therapeutic agents that can solve these aforementioned problems and can be used safely, without the side effects of drugs. In particular, there is a stronger need for research and development (R\&D) on nextgeneration antimicrobial materials with selective effects on oral bacteria [1]. There has been a growing interest of late in natural products for the prevention of infectious diseases in the oral cavity, and the interest in natural antimicrobial substances has further heightened due to the increased resistance to antibiotics [7].

Essential oils are volatile substances that are physically separated from scented plants. In general, essential oils are named after the plant from which they are extracted, and they have been called "essential oils" because they are considered to possess the essence of such plants' taste and fragrance [1]. Since recently, however, the antimicrobial, anti-inflammatory, antifungal, antiviral, and anti-cancer effects of essential oils have been reported, and they have been applied to various industrial fields, such as animal feeds, insecticides, dental products, and alternative medicine [8].

This study aimed to investigate the antimicrobial activity of essential oils against $C$. albicans, which causes infectious diseases in the oral cavity, by applying the essential oils lemon, tea tree, geranium, peppermint, and myrrh, and to provide fundamental data for oral health promotion by demonstrating the possibility of the use of essential oils as preventive and therapeutic agents for oral diseases. 


\section{Materials and Methods}

\section{Use strain}

C. albicans (KCTC 7965/ATCC 10231) was grown in yeast mold broth (YM, Difco, USA) and cultivated at $37^{\circ} \mathrm{C}$ for $24 \mathrm{~h}$. Five essential oils-lemon, tea tree, geranium, peppermint, and myrrh-refined in Caput, UK were purchased through DongSung Science.

\section{Antibacterial activity measurement}

To determine the antibacterial effect of each of the aroma essential oils that were used in this study, the paper disk agar measurement method and an $8 \mathrm{~mm}$ filter paper disk (Advantec Toyo Kaisha, Ltd.) were used. The disk was made to absorb $100 \mu \mathrm{l}$ of each aroma essential oil, after which the disk was anaerobically incubated at $37^{\circ} \mathrm{C}$ for $24 \mathrm{~h}$. The diameter of the clear zone $(\mathrm{mm})$ was measured to examine the antimicrobial activity of each aroma essential oil.

\section{Results}

The antibacterial effect of each aroma essential oil on $C$. albicans was examined by measuring the clear zone (Figure 1). The $C$. albicans clear zones were found to be $30 \mathrm{~mm}$ for lemon, $19 \mathrm{~mm}$ for tea tree, $20 \mathrm{~mm}$ for geranium, $19 \mathrm{~mm}$ for peppermint, and $21 \mathrm{~mm}$ for myrrh. Lemon oil showed the highest antimicrobial effect.

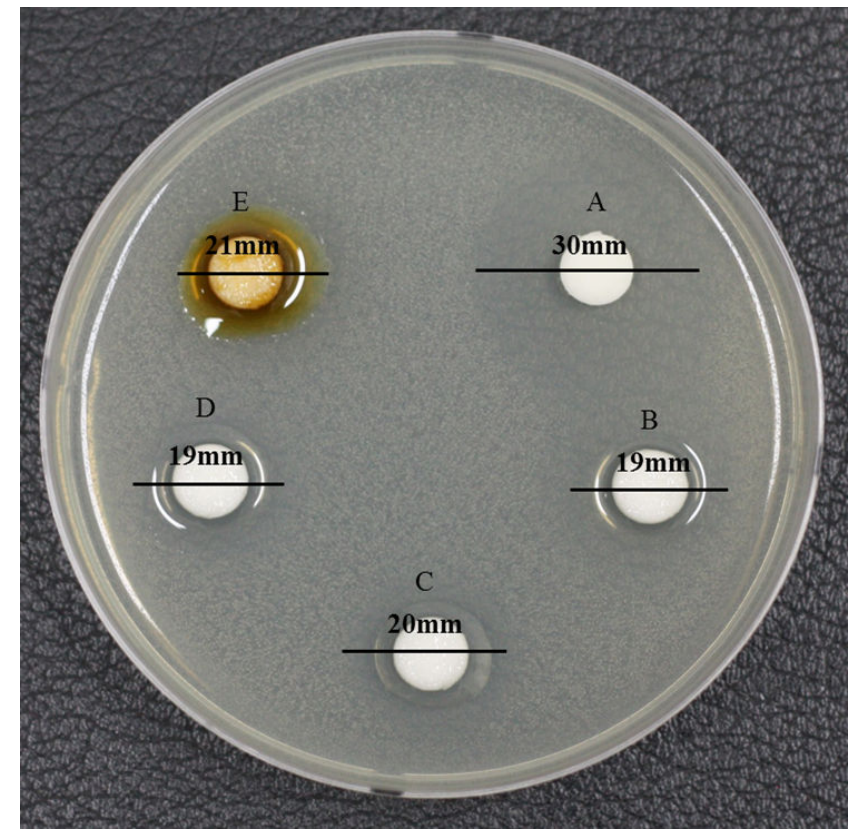

Figure 1. Clear zone of each essential oil against C. albicans. A: lemon; B: tea tree; C: geranium; D: peppermint; E: myrrh.

\section{Discussion}

C. albicans, the typical bacteria causing candidiasis, is the most common fungal pathogen present in the oral cavity [9]. To eliminate $C$. albicans and other oral-disease-causing bacteria from the human oral cavity, substances that can replace antibiotics are currently being developed, and there is an increasing interest in medicinal plants that can be easily recognized as natural products by consumers and that have excellent physiological activity [10].

Aroma essential oils are organic compounds with the inherent biochemical components of plants that promote natural healing, such as plant propagation and growth, protection against insects, and disease and wound healing [11]. Generated in the secondary metabolism process of plants and with unique aromas and functions, they consist of various combinations of several dozens to hundreds of natural chemical components [12]. Studies on the antimicrobial effect of plant extracts or essential oils have been actively conducted of late, and natural antimicrobial substances that can be used for preventing or inhibiting the progress of these diseases have been reported [13]. Essential oil components are known to interfere with the enzymatic activity of cells, destroying bacterial cell walls and killing bacteria [14]. They have also been reported to block clusters of Gram-positive bacteria, slow down bacterial replication, and remove endotoxin from Gram-negative bacteria to reduce oral pathogenic diseases [15]. Among the various essential oils in existence, lemon is widely used as a fragrance additive for spices, soaps, detergents, and perfumes. It has a healing power against preservatives, astringents, sterilizers, decontaminants, insecticides, and tonic and is used as an astringent to prevent excessive sebum production [16]. Tea tree is non-toxic and non-irritant, with 12-times-stronger preservative and sterilizing effects, is effective against various bacterial diseases, such as bacteria, virus, and bacterial infections, and has a particularly excellent effect on acne and athlete's foot [12]. Peppermint was first used as a whitening ingredient; later, it was used as a raw material for mouthwash to remove bad breath due to smoking [17]. Geranium is used for the treatment of diseases like eczema and psoriasis [18] and has antimicrobial effects against Staphylococcus aures, Bacillus subtilis, etc. [19]. Myrrh has antimicrobial and antiinflammatory effects and is used externally for treating inflammatory diseases like eczema [20].

Essential oils have been steadily studied in relation to causative organisms of dental caries and periodontal diseases [21,22], but there have been few studies on C. albicans. In this study, five essential oils were applied to $C$. albicans, a causative organism of candidiasis, and their antimicrobial effects were verified. As a result, a broad antimicrobial effect of $30 \mathrm{~mm}$ was shown by lemon, meaning that it effectively inhibited the growth of bacteria, followed by $21 \mathrm{~mm}$ for myrrh, $20 \mathrm{~mm}$ for geranium, $19 \mathrm{~mm}$ for tea tree, and $19 \mathrm{~mm}$ for peppermint. The aforementioned aroma essential oils thus showed excellent antimicrobial effects on $C$. albicans, a causative organism of candidiasis, an oral infectious disease, with lemon showing the highest antimicrobial activity. It is thus expected that these essential oils will be developed as the next-generation antimicrobial materials for inhibiting candidiasis. 


\section{Conclusion}

Essential oils were found to have excellent antimicrobial activity particularly against candida albicans (C. albicans), through their inhibition of its growth. Essential oils can thus be developed and widely used as agents for the prevention and treatment of oral infectious diseases.

\section{References}

1. Lee SY, Kim JG, Back BJ, Yang YM, Lee GY, Lee YH, Lee MA. Antimicrobial effect on essential oils on oral bacteria. J Korean Acad Pediatr Dent 2009; 36: 1-11.

2. Kim CS, Choi YK. Survey of adults perceptions of the association between chronic diseases and oral health. J Korean Dent Hyg Sci 2017; 17: 12-19.

3. Mizugai H, Isogai E, Hirose K, Chiba I. Effect of denture wearing on occurrence of candida species in the oral cavity. J Appl Res 2007; 7: 250-254.

4. Ashman RB, Farah CS, Wanasaengsakul S, Hu Y, Pang G, Clancy RL.Innate versus adaptive immunity in Candida albicans infection. Immunol Cell Biol 2004; 82: 196-204.

5. Williams D, Lewis M. Pathogenesis and treatment of oral candidosis. J Oral Microbiol 2011; 3: 1-11.

6. Cannon RD, Holmes AR, Mason AB, Monk BC. Oral Candida: clearance, colonization, or candidiasis? J Dent Res 1995; 74: 1152-1161.

7. Pai MR, Acharya LD, Udupa N. Evaluation of antiplaque activity of Azadirachta indica leaf extract gel-a 6-week clinical study. J Ethnopharmacol 2004; 90: 99-103.

8. DadaliogluI, Evrendilek G. Chemical compositions and antibacterial effects of essential oils of Turkish oregano (Origanum minutiflorum), bay laurel (Laurus nobilis), Spanish lavender (Lavandula stoechas L.), and fennel (Foeniculum vulgare) on common foodborne pathogens. J Agric Food Chem 2004; 52: 8255-8260.

9. Lamey, PJ, Samaranayake, LP. Oral candidosis: clinicopathological aspects. Dent Update 1988; 227-231.

10. Chen HL, Li DF, Chang BY, Gog LM, Dai JG, Yi GF. Effect of chinese herbal polysaccharides on the immunity and growth performance of young broilers. Poult Sci 2003; 82: 364-379.

11. Park EJ, Song YA, Song YM, Lee YJ. Aromatherapy begineer,s book for the therapist. Design Hub 2011; 11-99.

12. Jeong EJ, Kim JD. A study on the utilization and effect of essential oil. J Korean Soc Cosmet Cosmetol 2011; 1: 93-106.
13. Takarada K, Kimizuka R, Takahashi N, Honma K, Okuda $\mathrm{K}$, Kato T. A comparison of the antibacterial efficacies of essential oils against oral pathogens. Oral Microbiol Immunol 2004; 19: 61-64.

14. Ouhayoun JP. Penetrating the plaque biofilm: impact of essential oil mouthwash. J Clin Periodontol 2003; 30: 10-12.

15. Alviano WS, Mendonca-Filho RR, Alviano DS. Antimicrobial activity of Croton cajucara Benth linaloolrich essential oil on artificial biofilms and planktonic microorganisms. Oral Microbiol Immunol 2005; 20: 101-105.

16. Kim YM. A study on utilization and satisfaction in using aromatic products. Sookmyung Womens University Masters thesis 2008; 1-115.

17. Kim ES. The aromatherapy using aroma is a naturefriendly alternative therapy and has an effect on the physical, spiritual and emotional state. Konkuk University Masters thesis 2010; 1-66.

18. Bowles J. The basic chemistry of aromatherapeutic essential oils. Publisher: Allen \& Unwin Academic, Australia 1993; 1-236.

19. Seenivasan P, Manickkam J, Savarimuthu L. In vitro antibacterial activity of some plant essential oils. BMC Complement Alterm Med 2006; 6: 39.

20. Im RJ. Encyclopedia of Chosun medicinal plant III. Hankukmoonwhasa, Seoul, Korea 1997; 148.

21. Shapiro S, Meier A, Guggenheim B. The antimicrobial activity of essential oils and essential oil components towards oral bacteria. Oral Microbiol Immunol 1994; 9: 202-208.

22. Botelho MA, Nogueira NA, Bastos GM, Fonseca SG, Lemos TL, Matos FJ, Montenegro D, Heukelbach J, Rao VS, Brito GA. Antimicrobial activity of the essential oil from Lippia sidoides, carvacrol and thymol against oral pathogens. Braz J Med Biol Res 2007; 40: 349-356.

\section{*Correspondence to}

Young-Soon Choi

Department of Nursing

College of Health Science

Kangwon National University

Republic of Korea 